\title{
Multi-scale atmospheric environment modelling for urban areas
}

\author{
A. A. Baklanov ${ }^{1}$ and R. B. Nuterman ${ }^{1,2}$ \\ ${ }^{1}$ Danish Meteorological Institute, Copenhagen, Denmark \\ ${ }^{2}$ Tomsk State University, Tomsk, Russia
}

Received: 18 January 2009 - Revised: 22 March 2009 - Accepted: 2 April 2009 - Published: 15 April 2009

\begin{abstract}
Modern supercomputers allow realising multi-scale systems for assessment and forecasting of urban meteorology, air pollution and emergency preparedness and considering nesting with obstacle-resolved models. A multi-scale modelling system with downscaling from regional to city-scale with the Environment HIgh Resolution Limited Area Model (Enviro-HIRLAM) and to micro-scale with the obstacle-resolved Microscale Model for Urban Environment (M2UE) is suggested and demonstrated. The M2UE validation results versus the Mock Urban Setting Trial (MUST) experiment indicate satisfactory quality of the model. Necessary conditions for the choice of nested models, building descriptions, areas and resolutions of nested models are analysed. Two-way nesting (up- and down-scaling), when scale effects both directions (from the meso-scale on the micro-scale and from the micro-scale on the meso-scale), is also discussed.
\end{abstract}

\section{Introduction}

Due to increasing supercomputer power modern nested numerical meteorological and air pollution models realise model nesting/down-scaling from the global to the local scale and approach the necessary horizontal and vertical resolutions to provide weather and air quality forecasts for urban and local scales. However, most of such studies and systems consider down-scaling with the same type of mesoscale models (Grell et al., 1994; Jacobs et al., 1995) and only a few studies start considering the nesting of different types of models (e.g. meso-meteorological and micro-scale obstacle-resolved models, Kunz et al., 2000).

Atmospheric processes on the micro-meteorological scale depend not only on the local features, but also on larger scale processes, e.g. those of the meso-meteorological or even regional scales. Micro-meteorological and dispersion models for inhomogeneous areas, like urban domains, are sensitive to the choice of boundary conditions. In many research models and test studies the boundary conditions are simplified or artificial, mostly based on the assumptions of horizontal homogeneities in corresponding directions on the inlet and outlet boundaries of the considered domain.
However, in most of the urban simulations for real conditions only a small part of the urban area is considered in a micro-meteorological model and urban heterogeneities outside the simulation domain affect the micro-scale processes. Therefore, it is important to build a chain of models of different scales with nesting of high resolution models into larger scale lower resolution models.

Different requirements should be considered for the main key parameters and levels of parameterisations for urban models of different scales. Usually, the micro-scale (street canyon) models are obstacle-resolved and consider a detailed geometry of the buildings and the urban canopy, whereas the up-scaled city-scale (sub-meso) or meso-scale models consider parameterisations of urban effects or statistical descriptions of the urban building geometry.

In this paper we consider a multi-scale atmospheric environment modelling approach for urban areas with up- and down-scaling with different types of models from meso- and city-scale with parameterisations of sub-grid urban effects to the local- and micro-scales with the obstacle-resolved approach. 


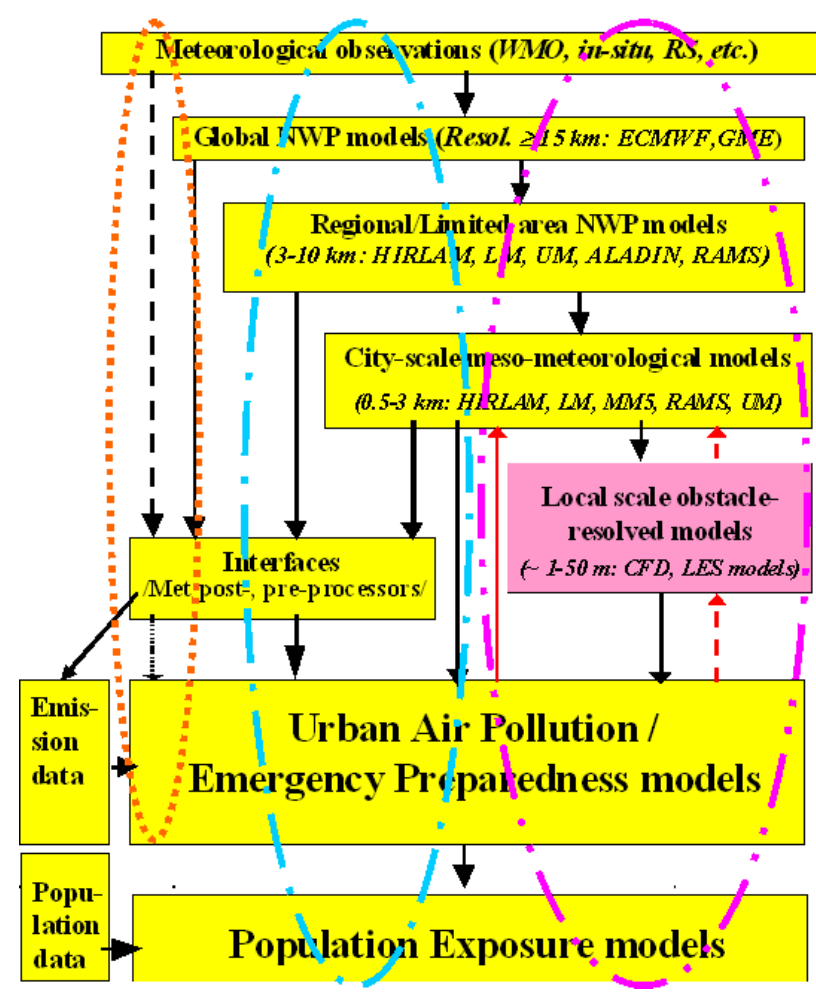

Figure 1. Model nesting in Urban Air Quality Information and Forecasting Systems: red $\ldots=$ common regulatory models; blue -.-.- = FUMAPEX multi-scale systems; pink -..-..- = new suggested down-scaling with obstacle-resolved models.

\section{Suggested methodology and models}

The methodology and several examples of realisation for such model-downscaling and integration for urban meteorology, air pollution and population exposure modelling were suggested recently in the EC FP5 project devoted to Integrated Systems for Forecasting Urban Meteorology, Air Pollution and Population Exposure (EMS-FUMAPEX, 2005; see also: http://fumapex.dmi.dk). This way included downscaling from regional (or global) meteorological models to the urban-scale meso-meteorological models with statistically parameterised building effects. As a next step, on-line integration considering two-way feedbacks between meteorology and pollutants was realised (Baklanov et al., 2008). Here in this paper further downscaling to the microscale with obstacle-resolved Computational Fluid Dynamics (CFD)-type models is suggested and considered. Figure 1 demonstrates the above mentioned schemes of the modeldownscaling in Urban Air Quality Information and Forecasting Systems (UAQIFS).

For nesting from the regional- to meso- and city-scale the Enviro-HIRLAM online integrated Numerical Weather Prediction - Atmospheric Chemistry Transport (NWP-ACT) model is used (Baklanov et al., 2008; Korsholm et al., 2008).
Several levels of urban parameterisation are considered in the model, they could be chosen depending on considered scales: for regional scale urban parameterisations are based on the roughness and flux corrections approach (EMS-FUMAPEX, 2005); for urban-scale on the building effects parameterisation, BEP (Martilli et al., 2002).

For local- and micro-scale nesting the Micro-scale Model for Urban Environment (M2UE) model is realised. This is a comprehensive CFD-type obstacle-resolved urban windflow and dispersion model (based on the Reynolds averaged Navier-Stokes approach and two-equation $k-\varepsilon$ turbulence closure), the M2UE, developed in cooperation with the Tomsk State University (Nuterman et al., 2008). For microscale M2UE modelling in this study we are focusing on dynamical processes. Because of the absence of the analysis of radiation/thermal effects in the MUST experiment; radiation processes are not included here, but could be considered in the model.

Boundary and initial conditions for the nested M2UE model are used from the Enviro-HIRLAM or other larger scale model simulations with corresponding interpolation keeping the mass-consistency. The choice of the boundary and initial conditions for the inner nested model is an important issue. For the boundaries a kind of Dirichlet condition usually is chosen to provide the values with an interpolation from the coarse to the fine grid. However, caution is advised in such an approach to keep the mass/energy consistence, otherwise a possible inconsistence can generate "parasite" waves or explode the model in long simulations. As one of the possible ways to diminish this problem, more soft conditions on one of the outlet boundaries can be chosen (however, paying attention to the fact that the Neumann type of boundary conditions will increase the computation time).

In some cases, when we switch from the roughness to the obstacle-resolved approach in the nested model, the interpolation procedure can be difficult for meteo-fields inside buildings and will lead an increase of the computation time due to necessary additional iterations. In such cases, as a possible alternative, the perturbation approach can be recommended, where the main meteorological variables are considered as a sum of two components: background (large-scale) values, described by the coarse-resolution model, and perturbations due to micro-scale features, described by the nested fine resolution model.

In a general sense, the scale interaction can play an important role in both directions: i.e. not only from a larger scale to the smaller micro-scale, but also from the urban/microscale to larger scale processes (e.g. atmospheric transport of harmful pollutants, initially released and dispersed in a street canyon; urban climate and wind climatology, etc.).

Therefore, two main types of nesting techniques for the model down-/up-scaling can be chosen: (i) one-way nesting, when effects of the local/micro-scale on the larger scale are not considered, and (ii) two-way nesting, when the scale effects in both directions (from the meso-scale on the 

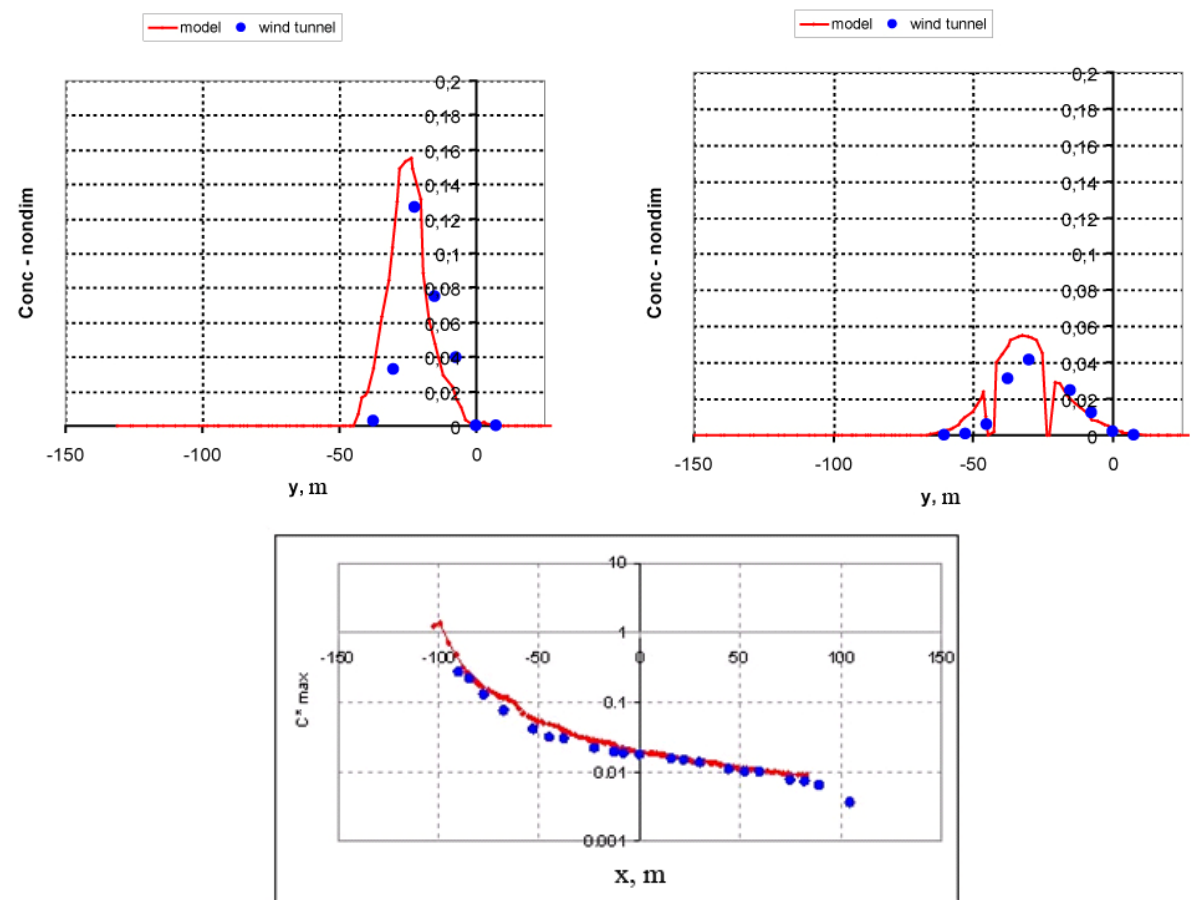

Figure 2. M2UE model validation versus the MUST (Di Sabatino et al., 2009): Profiles of concentration (upper graphs) on different distances from source: $27 \mathrm{~m}$ (left) and $50 \mathrm{~m}$ (right); and maximum values of concentration along axis $O x$ (lowest graph).

micro-scale and from the micro-scale on the meso-scale) are considered. In the last case both domains are usually run simultaneously to enable the feedbacks, and the terrain in the overlapping areas must be comparative to avoid mass inconsistency and generation of numerical noise.

\section{Results and discussion}

M2UE was tested for downscaling for urban air pollution and emergency preparedness modelling in combination with the Enviro-HIRLAM meso-scale model. Results for the mesoand city-scale were considered in Baklanov et al. (2008), so here we concentrate only on downscaling studies with M2UE up to the street-scale and possible up-scaling to consider the street-scale effects on wind-flow and pollutant transport on the local- and city-scales.

Figure 2 shows results of the M2UE model validation versus the Mock Urban Setting Trial (MUST) (Di Sabatino et al., 2009). The main point of the MUST is to simulate the real urban obstacles (with the use of non-regularly arranged 120 containers) and to measure the flow parameters/scalar transport in case of different meteorological conditions. Profiles of non-dimensional concentration $\left(C^{*}=C \cdot U_{\text {ref }} H_{\text {ref }}^{2} / Q\right.$, where $C$ denotes concentration, $U_{\text {ref }}=5.5 \mathrm{~m} / \mathrm{s}, H_{\text {ref }}=8.78 \mathrm{~m}$ and $Q$ is volume source) on different distances from the source (upper graphs): $27 \mathrm{~m}$ (left) and $50 \mathrm{~m}$ (right) show that the model describes well the concentration fields between buildings as close to the release site (where models with a statistical description of urban features usually fail) as well as with increasing distance from the site (see maximum values of concentrations along axis $O x$ on the lowest graph of Fig. 2). The maximum values of concentration (from $-100 \mathrm{~m}$ till $0 \mathrm{~m}$ ) decrease intensively with a parabolic dependence and then downstream (from $0 \mathrm{~m}$ till $100 \mathrm{~m}$ ) the function becomes almost linear. Thus, there is a maximum of concentrations along the basic flow direction which is moved due to the complex orography of the computational domain (Fig. 2, lowest graph). The simulations with different turbulence closures show, that even with a high sensitivity of the turbulence kinetic energy in the canopy layer, there is no substantial effect of the turbulence schemes on the pollution dispersion properties of the particular CFD model (Di Sabatino et al., 2009; Nuterman et al., 2008). For distances longer than 3-4 buildings from the release the statistical description of the building structure (Martilli et al., 2002) gives suitable results, therefore $\mathrm{M} 2 \mathrm{UE}$ with a more precise (and expensive) obstacle-resolved approach is needed only for the first 2-4 buildings from the source. The output from the micro-scale simulation is used for meso-scale runs with averaged characteristics by the BEP module. As an example, Fig. 3 shows the vertical profile of the averaged velocity component (a) provided by M2UE, a vertical profile of the drag coefficient (b), which is a very important input parameter for the city-scale model; the kinetic energy of turbulence (c), and the dispersive kinetic energy (d). 

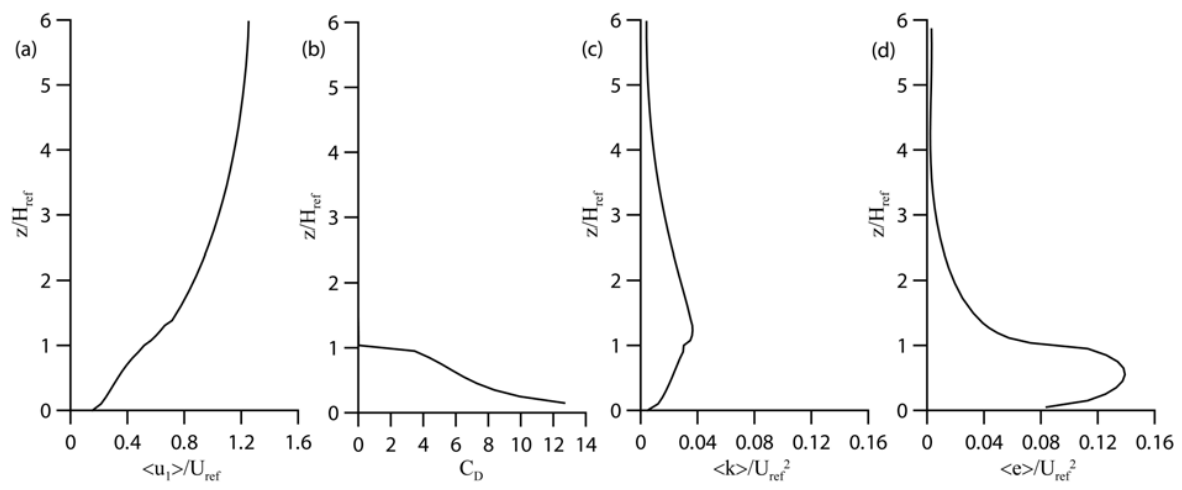

Figure 3. Vertical profiles of averaged variables: (a) mean axial velocity; (b) drag coefficient (non-dimensional); (c) kinetic energy of turbulence; (d) dispersive kinetic energy.

Let us discuss the computation of the dispersive kinetic energy, which is the kinetic energy due to the time-averaged structures smaller than a grid cell (Martilli et al., 2007). It means that for the calculation of the dispersive kinetic energy we consider the grid cell containing one obstacle with sub-grid deterministic motions within the cell. The same approach is used for the computation of the drag coefficient.

The results of averaging (Fig. 3) show that there is no logarithmic law for $u_{1}$ at the roof level and above the array of containers. The drag coefficient is not a constant and it is substantially varying with height. It does not mean that there is not any similarity of such a variation for different obstacles, but the values of $C_{D}$ depend on the geometry of the obstacles and the flow conditions. The simulations show that the dispersive kinetic energy is substantially higher than the turbulent kinetic energy in the canopy. It stresses the importance of a correct parameterization of dispersive stresses in larger scale models because they have a significant effect on the total gradient of sub-grid fluxes.

One of the important aspects in the model nesting is the necessary scale ratio (between the grid resolutions of the main and the nested models) to keep the numerical stability, suitable approximation and accuracy of the models. Our results and experience of other modellers show that the ratio should be not higher than 3-5.

Let us consider an example showing the importance of not only down-scaling to the street-scale, but also up-scaling for local- or city-scale modeling of releases in an urban environment. Figure 4 shows the considered model down-scaling for a selected area of Copenhagen with the simulation of meteorological fields and air pollution for the two largest areas with the Enviro-HIRLAM model with a statistical description of urban parameters and for the inner smaller area by M2UE resolving the real building structure and using boundary conditions from the larger area simulations. For a release position sensitivity study two separate runs are considered, where the release position is moved only by $10 \mathrm{~m}$ (positions 1 and 2 in Fig. 4): the first case (position 2, Fig. 5, right) considers

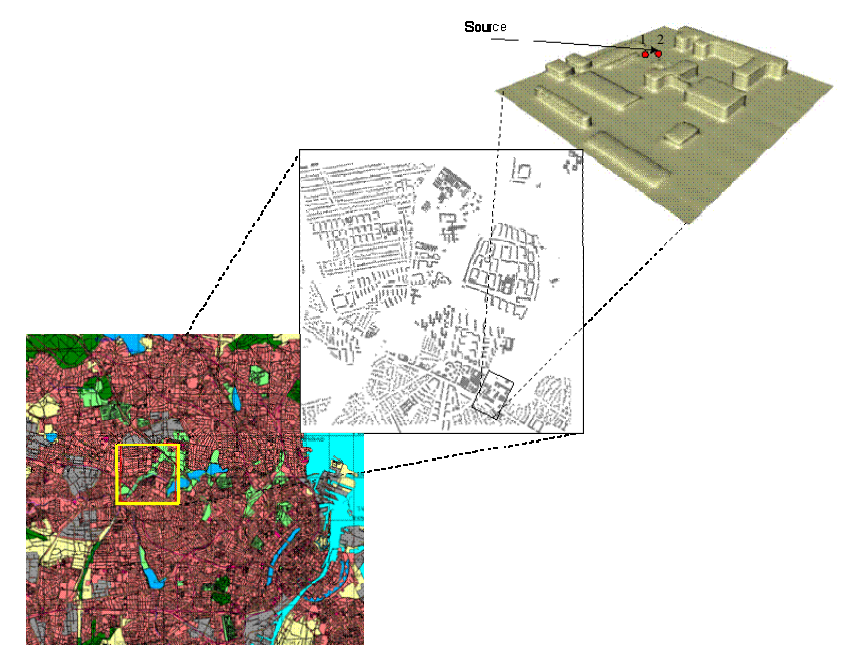

Figure 4. Micro-scale Model for Urban Environment (M2UE) downscaling for the selected Copenhagen area. The release positions 1 and 2 are shown at the inner domain.

the release in the main flow between 2 buildings, the second case (position 1, left) in an aerodynamic shadow close to the building. In Fig. 5 near surface velocity fields and iso-surfaces of concentrations for both release positions are presented (left and right). The analysis of the results shows that this small variation of the release site leads to a great change of the plume dispersion: in the first case it is transported quickly between the buildings by the main streams to the outlet border, but in the second case it turned to the right and is much more slowly transported through the other street canyon to the right border of the modelling area. So, further up-scale simulations for these cases show very different speeds of plume transport (which is extremely important in a case of emergency preparedness) and patterns of contaminant concentration and deposition. It is clear that without such a nesting with an obstacle-resolved model it is not possible to describe this effect. However, it is important to stress 

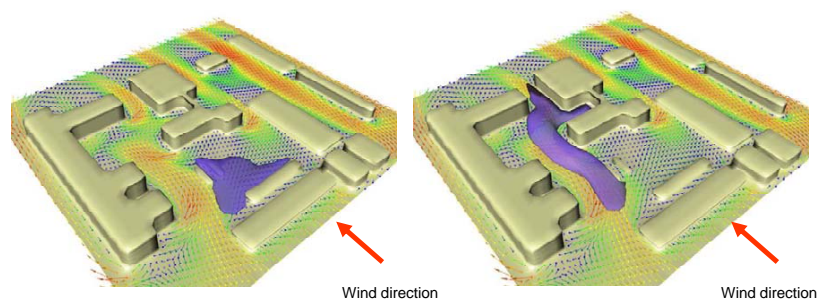

Figure 5. M2UE release position sensitivity study for the Copenhagen area. Near surface velocity field and iso-surfaces of concentration: different release position (position 1 - left and position $2-$ right).

that two-way nesting is not always reasonable to consider, because this approach is more expensive in comparison with one-way nesting. Therefore, for the considered specific problems it is recommended to do in advance (before suggesting the modelling system for end-users) a sensitivity study of the possible feedbacks from the micro-scale to larger scale processes.

\section{Conclusions}

Modern supercomputers allow realising multi-scale systems for assessment and forecasting of urban meteorology, air pollution and emergency preparedness and considering downscaling with obstacle-resolved models. Such models can be used not only for advanced planning safety measures, postaccidental analysis or health/environment impact assessment modelling, but also in the nearest future for operational forecasting or emergency preparedness modelling in cases of terror acts, accidental releases, fires, etc.

Multi-scale modelling with one-way nesting for model downscaling (regional to city-scale Enviro-HIRLAM and micro-scale obstacle-resolved M2UE) is suggested and demonstrated. The M2UE validation results versus the MUST experiment indicate satisfactory quality of the model.

Close to release/emission sources in urban areas it is often (e.g. emergency preparedness) important to consider the real geometry of the nearest 2-3 buildings; for larger scale dispersion the statistical description of urban characteristics is enough. It is recommended that the resolution of nested models should not be more than 3-5 times higher than that of the previous model.

Two-way nesting (up- and down-scaling), when scale effects in both directions (from the meso-scale on the microscale and from the micro-scale on the meso-scale) are considered, is also possible in the suggested multi-scale modelling system, but is more expensive and needs further analysis.
Acknowledgements. The study is funded by the EC FP7 project MEGAPOLI (grant no. 212520), Danish Council for Strategic Research (CEEH) and INTAS grant no. 06-1000016-5928, and linked with FUMAPEX, COST732 and 728 studies.

Edited by: P. J. H. Builtjes

Reviewed by: A. Ebel and another anonymous referee

\section{References}

Baklanov, A., Korsholm, U., Mahura, A., Petersen, C., and Gross, A.: ENVIRO-HIRLAM: on-line coupled modelling of urban meteorology and air pollution, Adv. Sci. Res., 2, 41-46, 2008.

Di Sabatino S., Buccolieri, R., Olesen, H., Ketzel, M., Berkowicz, R., Franke, J., Schatzmann, M., Schlunzen, H., Britter, R., Borrego, C., Costa, A. M., Castelli, S. T., Reisin, T., Hellsten, A., Saloranta, J., Moussiopoulos, N., Barmpas, F., Brzozowski, K., Goricsan, I., Balczo, M., Bartzis, J., Efthimiou, G., Santiago, J. L., Martilli, A., Piringer, M., Hirtl, M., Baklanov, A., Nuterman, R., and Starchenko, A.: COST 732 in practice: the MUST model evaluation exercise, Int. J. Environ. Pollut., in press, 2009.

EMS-FUMAPEX: Urban Meteorology and Atmospheric Pollution, edited by: Baklanov, A., Joffre, S., and Galmarini, S., Special Issue, Atmos. Chem. Phys., http://www.atmos-chem-phys.net/ special_issue24.html, 2005.

Grell, G. A., Dudia, J, and Stauffer, D. R.: A description of the fifth generation Penn State/NCAR Mesoscale Model (MM5), NCAR, Tech. Note NCAR/TN-398 + STR, 1994.

Jacobs, H. J., Feldmann, H., Hass, H., and Memmesheimer, M.: The use of nested models for air pollution studies: An application of the EURAD model to a SANA episode, J. Appl. Met., 34, 13011319, 1995.

Korsholm, U. S., Baklanov, A., Gross, A., and Sørensen, J. H.: On the importance of the meteorological coupling interval in dispersion modeling during ETEX-1, Atmos. Environ., doi:10.1016/j.atmosenv.2008.11.017, 2008.

Kunz, R., Khatib, I., and Moussiopoulos, N.: Coupling of mesoscale and microscale models - an approach to simulate scale interaction, Environ. Modell. Softw., 15, 597-602, 2000.

Martilli, A., Clappier, A., and Rotach, M. W.: An Urban Surface Exchange Parameterisation for Mesoscale Models, Bound.-Lay. Meteorol., 104, 261-304, 2002.

Martilli, A. and Santiago, J. L.: CFD simulation of airflow over a regular array of cubes. Part II: analysis of spatial average properties, Bound.-Lay. Meteorol., 122, 635-654, 2007.

Nuterman, R. B., Starchenko, A. V., and Baklanov, A. A.: Development and evaluation of a microscale meteorological model for investigation of airflows in urban terrain, J. Computational Technologies, 13(3), 37-43, 2008. 